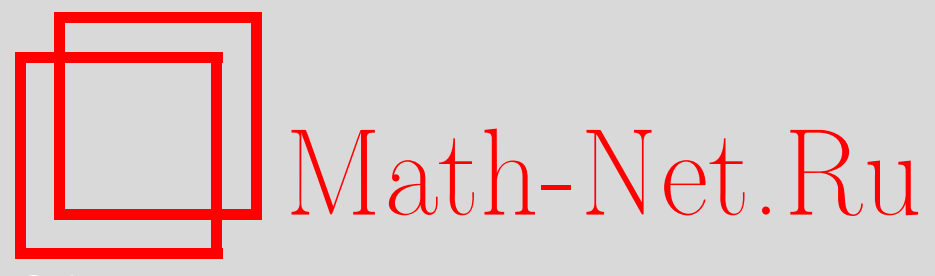

С. Дворянинов, Закон Архимеда и закон сохранения энергии, Квант, 2021, номер 5, 41-43

DOI: https://doi.org/10.4213/kvant20210505

Использование Общероссийского математического портала Math-Net.Ru подразумевает, что вы прочитали и согласны с пользовательским соглашением http://www.mathnet.ru/rus/agreement

Параметры загрузки:

IP : 3.89.197.203

26 апреля 2023 г., 13:44:35

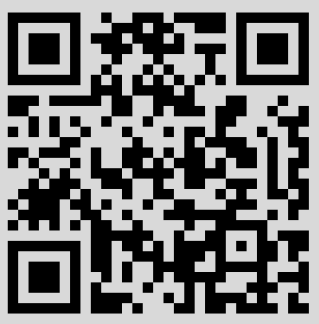




\section{Закон Архимеда и закон сохранения энергии}

\section{С.ДВОРЯНИНОВ}

Рассмотрим такую задачу. Пусть имеется полый внутри кубик с длиной ребра $a$, грани которого сделаны из прочной и очень тонкой фольги. Этот кубик плавает на поверхности воды так, что четыре его ребра вертикальны (рис.1). Кубик сместили вниз по вертикали

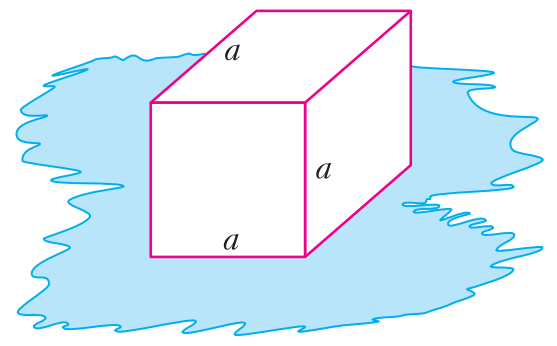

PИC. 1

на расстояние $x$, при этом часть его оказалась в воде. Считая массу кубика равной нулю, надо найти совершенную при этом работу.

Дать однозначный ответ к задаче о погружении кубика в воду нельзя. Оказывается, ответ зависит от того сосуда, в который налита вода. Обсудим несколько случаев.

\section{Бак заполнен водой до самого верха}

В этом случае при малейшем погружении нашего кубика вода будет из бака выливаться, но уровень воды в баке будет оставаться неизменным. От него и будет отсчитывать глубину погружения. Эта глубина $H_{1}$ соответствует расстоянию от уровня воды до нижней грани кубика и равна $x$ (рис.2). При этом через край бака выльется вода, объем которой $a^{2} x$ и масса $\rho a^{2} x$. Вначале центр масс этой воды находился на глубине $x / 2$, следовательно, работа, совершенная по подъему этой воды до верхнего края бака, равна

DOI: https://doi.org/10.4213/kvant20210505

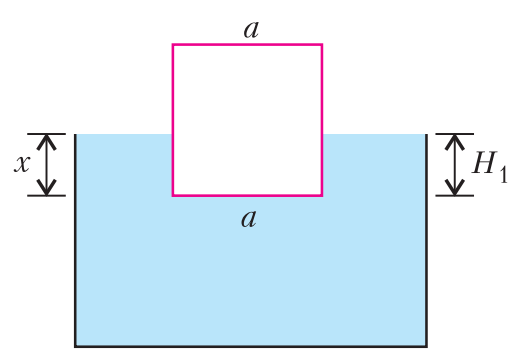

Puc. 2

приращению ее потенциальной энергии:

$$
A_{1}(x)=E_{\text {п1 }}=\rho a^{2} x \cdot g \cdot \frac{x}{2}=\frac{1}{2} \rho a^{2} g x^{2} .
$$

Сила, необходимая для погружения тела в воду, является переменной и зависит от глубины погружения, поэтому

$$
\int_{0}^{x} F_{1}(x) d x=\frac{1}{2} \rho a^{2} g x^{2} .
$$

Дифференцируя обе части этого равенства по $x$, находим

$$
F_{1}(x)=\rho a^{2} g x .
$$

Сила $F_{1}(x)$ - это сила, противодействующая выталкивающей силе, т.е. силе Архимеда $F_{\mathrm{A}}$. (Сила тяжести на наше тело не действует, оно невесомо.) Сила Архимеда направлена вверх и численно равна силе тяжести воды, вытесненной кубиком из бака. В повседневной жизни силу тяжести чаще называют весом. Заметим, что в данном случае вытесненная вода - эта вода, вылитая из бака. Давление со стороны воды на нижнюю грань кубика равно

$$
p(x)=\frac{F_{1}(x)}{S}=\frac{\rho a^{2} g x}{a^{2}}=\rho g x .
$$

Оно действует вверх.

Пусть глубина погружения стала равной $a$. При дальнейшем перемещении кубика вниз вода из бака выливаться не будет, сила
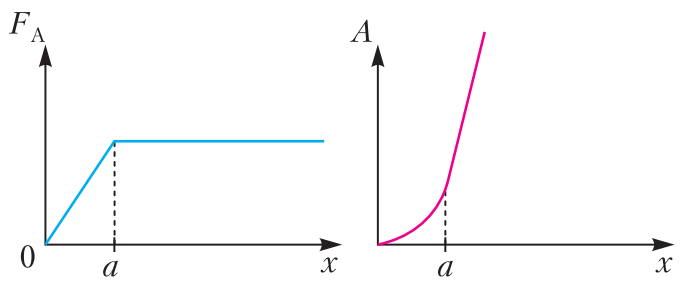

Pис. 3 
Архимеда теперь будет оставаться постоянной и равной $F_{\mathrm{A}}=\rho a^{3} g$. Графики на рисунке 3 показывают, как от глубины погружения зависят сила Архимеда и необходимая для ее преодоления работа.

Если сила Архимеда равна весу плавающего на поверхности воды тела (т.е. силе тяжести тела), то медленное перемещение тела вдоль горизонтали с малой скоростью требует малой силы. Если тело целиком находится в воде, то легко осуществить его перемещение и по вертикали. Каждый знает это из личного опыта: при выходе на берег после продолжительного плавания в море особенно заметно, как легко можно было двигать руками и ногами в воде по сравнению с необходимыми усилиями на суше.

Выражение для работы $A_{1}$ можно получить и по-другому. Ясно, что в нашем случае вода, заполняющая параллелепипед $a \times a \times x$, поднялась на уровень бака. Мысленно разделим всю воду на очень узкие вертикальные столбики, практически на материальные отрезки. Медленный поворот каждого столбика на $90^{\circ}$ и приведение его в горизонтальное положение не требует никакой работы. Но теперь получается, что каждую точку такого столбика-отрезка (т.е. весь отрезок целиком) надо поднять на одну и ту же высоту $x / 2$. Это равносильно тому, что на эту высоту надо поднять всю массу воды $\rho a^{2} x$. Для исходного параллелепипеда высота была переменной, своей для каждой точки.

\section{Вода из бака не выливается}

Рассмотрим бак с водой, имеющий форму параллелепипеда. Его основание - квадрат со стороной $a \sqrt{K}, K>1$, а высота бака большая, так что даже при полном погружении кубика в воду вода из бака не выливается.

Назовем начальный уровень воды в баке нулевым. Пусть под действием силы, приложенной к кубику и направленной вертикально вниз, кубик сместился вниз от нулевого уровня на расстояние $x$ и частично оказался в воде (рис.4). При этом кубик «вытеснил» воду, объем которой равен $a^{2} x$ и которая распределилась выше нулевого уровня. Теперь площадь поверхности воды стала равной $(K-1) a^{2}$. Пусть уровень воды в баке

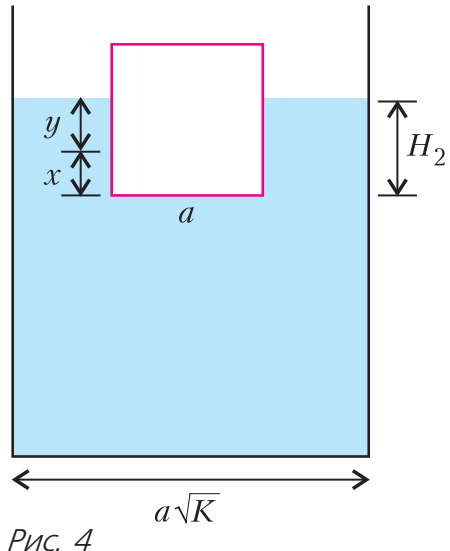

повысился на величину $y$. Согласно закону сохранения объема получаем, что $(K-1) a^{2} y=a^{2} x$, откуда

$$
y=\frac{x}{K-1} .
$$

При этом от нового уровня воды в баке кубик погрузился на глубину

$$
H_{2}=x+y=\frac{K}{K-1} x .
$$

Пусть верхняя грань кубика в воду еще не попала, т.е.

$$
H_{2}=\frac{K}{K-1} x<a .
$$

Тогда центр масс вытесненной кубиком воды сместился вверх по вертикали на

$$
h=\frac{x+y}{2}=\frac{K}{2(K-1)} x .
$$

Потенциальная энергия вытесненной воды получила при этом приращение

$$
\begin{aligned}
E_{\text {п2 }}=m g h=\frac{1}{2} \rho a^{2} x \cdot g \cdot \frac{K}{K-1} & x= \\
= & \frac{1}{2} \rho a^{2} g \frac{K}{K-1} x^{2} .
\end{aligned}
$$

Именно такую работу

$$
A_{2}(x)=\frac{1}{2} \rho a^{2} g H_{2} x=\frac{1}{2} \rho a^{2} g \frac{K}{K-1} x^{2}
$$

необходимо совершить для погружения кубика в воду на глубину $x$ от начального уровня.

Работа $A_{2}$ зависит от $x$ квадратичным образом: например, увеличение $x$ в два раза требует вчетверо большей работы. С этим обстоятельством мы сталкиваемся на море или реке, когда пытаемся «утопить» резино- 


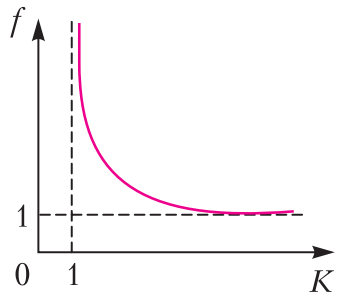

Рис. 5 вый мяч. А вот с зависимостью работы от параметра $K$, т.е. от площади «зеркала» бака, мы встречаемся нечасто. График функции $f(K)=$ $=\frac{K}{K-1}$ показан на

рисунке 5. На бесконечном интервале

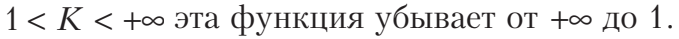

Пусть бак с водой очень-очень узкий, чуть шире кубика, т.е. $K \rightarrow 1+0$. В этом случае работа по погружению кубика в воду очень велика. На большом озере, при $K \rightarrow+\infty$, эта работа оказывается наименьшей из всех возможных и равной работе силы тяжести жидкости, вытесненной кубиком. При этом считается, что уровень воды в озере не изменился.

Приобретенная потенциальная энергия возникла в результате работы переменной силы. То, что сила переменная, знаем из опыта: чтобы глубже погрузить плавающее на поверхности воды тело, надо приложить большую силу. Следовательно,

$$
\int_{0}^{x} F_{2}(x) d x=\rho a^{2} g \frac{K}{2(K-1)} x^{2} .
$$

Дифференцируя, получаем

$$
F_{2}(x)=\rho a^{2} g \frac{K}{K-1} x .
$$

Очевидно, что

$$
F_{2}(x)=\rho a^{2} g \frac{K}{K-1} x>F_{1}(x)=\rho a^{2} g x .
$$

Это неравенство означает, что во втором случае сила, необходимая для погружения кубика на глубину $x$ от начального уровня, больше, чем в первом случае. Более того, эта требуемая сила может быть сколь угодно большой, если $K$ достаточно близко к единице, т.е. если бак с водой достаточно узкий. Именно это обстоятельство используется при создании гидравлических машин, в частности гидравлических прессов. В этом случае получается такой парадоксальный результат. Пусть наш кубик - это «корабль», а бак с водой - это «море». Тогда на этом корабле можно разместить сколь угодно тяжелый груз и корабль не утонет. Для этого надо взять параметр $K$ достаточно близким к

единице. Только вот плыть такому кораблю некуда - он буквально зажат со всех сторон «морскими берегами».

В заключение еще раз вспомним глубину погружения кубика в воду $H_{2}=\frac{K}{K-1} x$ и заметим, что на этой глубине на кубик действует сила

$$
F_{2}(x)=\rho a^{2} g H=\rho a^{2} H \cdot g .
$$

А это и есть сила Архимеда, равная весу воды, вытесненной кубиком. Подумайте, к какой части воды относится в данном случае прилагательное вытесненная и куда она вытеснена. Главная характеристика вытесненной воды - это ее объем, он всегда равен объему той части тела, которая находится в воде.

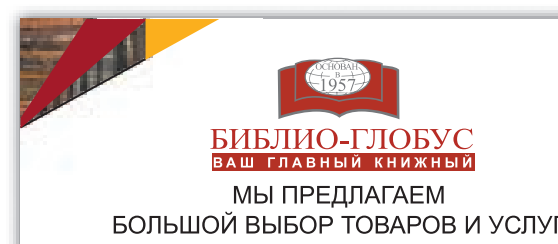

Услуги

॥ Интернет-магазин www.bgshop.ru

Kaфpe

- Клубные (дисконтные)

карты и акции

Подарочные карты

п Предварительные

заказы на книги

॥ Встречи с авторами

Читательские клубы

по интересам

Индивидуальное

обслуживание

П Подарочная упаковка

Доставка книг из-за рубежа

- Выставки-продажи
АССОРТИМЕНТ

- Книги

I Аудиокниги

I Антиквариат

и предметы

коллекционирования

І Фильмы, музыка,

игры, софт

I Канцелярские

и офисные товары

І Цветы

I Сувениры

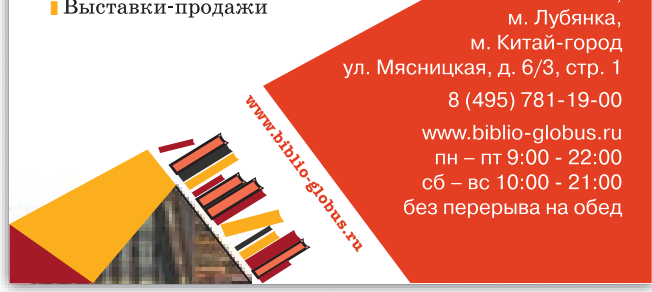

